\title{
Challenges of the New ICRP Eye Lens Dose Limit
}

\author{
Abdalla N Al-Haj ${ }^{{ }^{*}}$ and Aida Lobriguito ${ }^{2}$ \\ ${ }^{1}$ King Faisal Specialist Hospital \& Research Centre, Riyadh, Saudi Arabia \\ ${ }^{2}$ King Fahad Medical City, Riyadh, Saudi Arabia
}

"Corresponding author: Al-Haj AN, King Faisal Specialist Hospital \& Research Centre, PO Box 3354, Riyadh 11211, Saudi Arabia; Tel: 966505413157; Fax: 966114424777; E-mail: abdal@kfshrc.edu.sa

Received date: July 02, 2016; Accepted date: October 25, 2016; Published date: November 02, 2016

Copyright: (C) 2016 Al-Haj AN, et al. This is an open-access article distributed under the terms of the Creative Commons Attribution License, which permits unrestricted use, distribution, and reproduction in any medium, provided the original author and source are credited.

\section{Short Communication}

Radiation protection in the use of ionizing radiation in medicine is becoming more and more important because of its increasing use for imaging and therapy and because of complex procedures and technology. The US study showed that the population dose due to medical uses of ionizing radiation increased to about $49 \%$ of the total population radiation dose in year 2006 [1]. The increase is due to the wide use of medical man-made sources of radiation in computed tomography (CT) imaging, interventional radiology and nuclear medicine. Justification of medical exposures and optimization of patient and staff protection are the thrust of the International Atomic Energy Agency (IAEA) and other international communities and regulatory bodies.

A review of the International Commission on Radiological Protection (ICRP) Report No. 60 (1990 Recommendations of the ICRP) was started in 1999 for new updates, consolidation and additional protection guidance [2]. A new ICRP recommendation was released in year 2007 in its ICRP Report No. 103. One of the major changes in ICRP 60 is the new values for tissue weighting factors [3]. Based on epidemiological evidences and consultations, ICRP changed the annual occupational equivalent dose limit to the lens of the eye in 2011 from $150 \mathrm{mSv}$ to $20 \mathrm{mSv}$ averaged over 5 years but not to exceed $50 \mathrm{mSv}$ in a single year. This new dose limit was adopted by IAEA in its interim basic safety standards and in its final edition which was issued in 2014 [4]. IAEA also published TECDOC 1731 incorporating the new eye lens dose limit and the clinical implications of the change [5].

The new ICRP eye lens dose limit can have some impacts on clinical practices of interventional radiologists, cardiologists and positron emission tomography (PET) staff. Our study on occupational doses showed that staff involved in angiography and cardiac catheterization procedures in Saudi Arabia belong to the high dose category [6]. The study of Struelens showed that interventional radiology and cardiology eye lens doses could be in the range of $0.01 \mathrm{mSv}$ to $4 \mathrm{mSv}$ [7]. Vano et al. [8] specified that eye lens doses could reach $11 \mathrm{mSv}$ per procedure if typical protective tools are not used; about $0.4 \mathrm{mSv}$ when such tools are used; about $1 \mathrm{mSv}$ when personal dosimeter has not been regularly used. These figures are in agreement with the measured doses per procedure (1.1 mSv maximum) at the eye level over personal protective devices [9]. However, Holmberg et al. [10] reported that interventional radiologists and cardiologists can have annual eye lens doses in the range of $1 \mathrm{mSv}$ to $200 \mathrm{mSv}$ and this wide range is due to the large coverage of the study. In PET, the annual eye lens dose of the staff could be about $4 \mathrm{mSv}$ [11].

The drastic change in the eye lens dose limit brings challenges in the implementation. One of these challenges is the development of standard dosimetry and methods for dose monitoring. The work of
Carinou et al. [12] summarized the different reports on the calibration, protocols and conversion coefficients when using $\mathrm{Hp}(10)$ and $\mathrm{Hp}(0.07)$ operational quantities for estimating eye lens doses. They stated that calibration of dosimeters with photons using slab phantom and cylindrical phantom can give a difference in the eye lens dose of about $\pm 5 \%$ except for angles greater than 75 degrees for which the cylinder phantom can provide a better estimate. Following the meeting of the German Commission on Radiological Protection in February 2010, the Commission stated that the operational dose equivalent quantity $\mathrm{Hp}(0.07)$ adequately estimates the eye lens dose at energies less than $200 \mathrm{kev}$ and $\mathrm{Hp}(10)$ for energies greater than $100 \mathrm{kev}$. In addition, the operational quantity $\mathrm{Hp}(3)$ can accurately estimate the eye lens dose for beta radiation fields provided the dosimeter is placed very close to the lens of the eye to avoid underestimation [13]. A Monte Carlo modeling to determine the air kerma to $\mathrm{Hp}(3)$ conversion coefficient at a depth of $3 \mathrm{~mm}$ using energies from $10 \mathrm{kev}$ to $10 \mathrm{mev}$ was performed by Gualdrini et al. [14] to improve the overall quality of eye lens dose assessment procedures. The work of Vano et al. [8] on the use of a reference dosimeter placed in the $\mathrm{C}$-arm allows the assessment of the eye lens doses when the personal dosimeters are not frequently used but this would give an underestimation due to the different X-ray tube orientation.

The availability of different conversion coefficients using $\mathrm{Hp}(10)$, $\mathrm{Hp}(0.07)$ and $\mathrm{Hp}(3)$ for assessing eye lens doses does not lead to the conclusion of what the best dosimetry protocol is to be used. Development of $\mathrm{Hp}$ (3) dosimeters is underway but calibration and type testing are being standardized [15]. The development of a standard protocol for eye lens dose monitoring is a big task and challenge.

Another challenge is the clinical practices of interventional radiologists and cardiologists and PET/CT staff. Our study [16] on using the $\mathrm{Hp}(0.07)$ to estimate the eye lens doses of staff (cardiologists, nurses and technologists) involved in cardiac catheterization procedures in Saudi Arabia showed that cardiologists could approach or exceed the new dose limit (Figure 1). It showed that introduction of new clinical protocols and increase in patient workload can greatly increase the eye lens doses of cardiologists (Table 1). The knowledge gap on radiation safety was also a contributory factor for high eye lens doses. Our study on the occupational doses in PET/CT showed that the measured mean staff whole body dose is $12.4 \mu \mathrm{Sv}$ per procedure [17]. For a conservative number of 5 patients per day, the estimated annual whole body dose is $15 \mathrm{mSv}$ per year. Doubling the patient workload will increase the whole body dose by factor of about 2 and hence it is possible that PET/CT staff eye lens doses can approach or possibly exceed the new dose limit if workload is increased. The study showed the lack of training on radiation safety. 


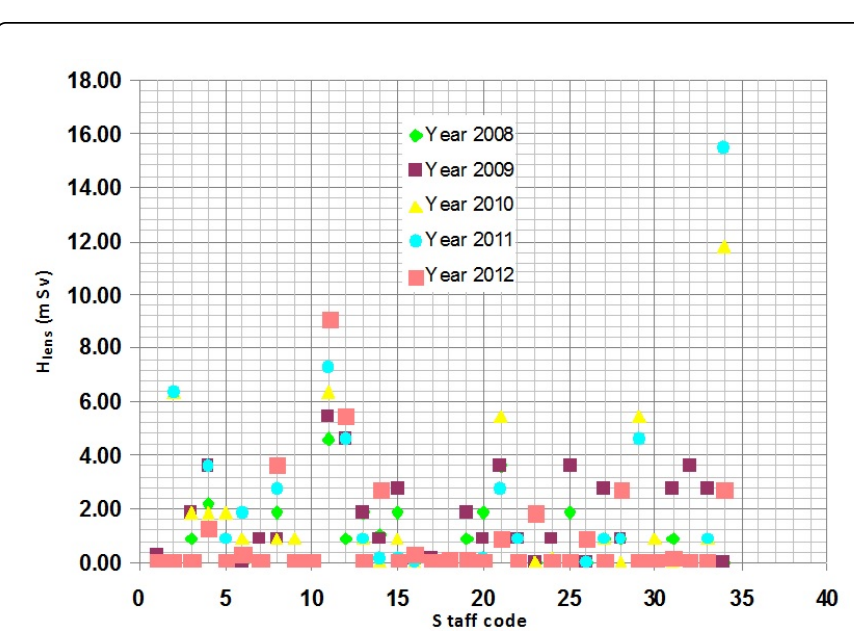

Figure 1: Scatter plot of the calculated eye lens doses from $\mathrm{Hp}(0.07)$ values for the five year monitoring period (2008-2012). The highest dose value belongs to a cardiologist.

\begin{tabular}{|l|l|l|l|l|l|}
\hline Category & Year 2008 & $\mathbf{2 0 0 9}$ & $\mathbf{2 0 1 0}$ & $\mathbf{2 0 1 1}$ & $\mathbf{2 0 1 2}$ \\
\hline Nurses & $0.28(0.45)$ & $1.21(1.33)$ & $0.82(1.68)$ & $0.83(1.38)$ & $0.83(1.38)$ \\
\hline Technologists & $0.63(0.78)$ & $1.2(1.24)$ & $0.29(0.57)$ & $0.17(0.50)$ & $0.17(0.50)$ \\
\hline Cardiologists & $1.36(1.61)$ & $1.82(2.11)$ & $3.88(3.52)$ & $4.21(4.37)$ & $2.12(0.50)$ \\
\hline
\end{tabular}

Table 1: Average eye lens dose (Hlens) in $\mathrm{mSv}$ of staff in different categories with the standard deviation. The increase in dose for years 2010 and 2011 is due to the introduction of new clinical protocols.

Although the use of protective tools is in place, knowledge of the radiation protection program can reduce the eye lens dose by $50 \%$ to $70 \%$. With this new eye dose limit, there is also a need to identify competencies of staff for the different procedures from simple to complex. When new procedures will be implemented, it is necessary to perform staff eye lens dose assessment using phantoms to simulate patients before the clinical use of the procedure and established protocol needs to be followed as part of the radiation protection program to reduce staff eye lens doses.

\section{References}

1. National Commission on Radiation Protection and Measurements (2009) Report No. 160, Ionizing radiation exposure of the population of the United States. Bethesda, MD, USA.

2. International Commission on Radiological Protection (1991) 1990 Recommendations of the International Commission on Radiological Protection. ICRP Publication 60. Ann ICRP 21: 1-3.
3. International Commission on Radiological Protection (2007) The 2007 Recommendations of the International Commission on Radiological Protection. ICRP Publication 103. Ann ICRP 37: 1-332

4. International Atomic Energy Agency (2014) Safety Standards Series No. GSR Part 3, Radiation protection and safety of radiation sources: International basic safety standards general safety requirements, Vienna, Austria.

5. International Atomic Energy Agency (2013) Implications for occupational radiation protection of the new dose limit for the lens of the eye. Vienna, Austria.

6. Al-Haj AN, Lagarde, Charlie S (2002) Statistical analysis of historical occupational dose records at a large medical center. Health Phys 83: 854-860.

7. Stuelens L (2013) Eye lens doses for medical staff performing interventional procedures. Radiation Protection Dosimetry and Calibration.

8. Vano E, Gonzales L, Fernandez JM, Haskai ZJ (2008) Eye lens exposure to radiation in interventional suites: Caution is warranted. Radiol 248: 945-953.

9. Vano E, Fernandez JM, Sanchez RM, Dauer LT (2013) Realistic approach to estimate lens doses and cataract radiation risk in cardiology when personal dosimeters have not been regularly used. Health Phys 105: 330-339.

10. Holmberg O, Czarwinski R, Mettler F (2010) The importance and unique aspects of radiation protection in medicine. European J Radiol 76: 6-10.

11. Guiu-Souto J, Sanchez-Garcia M, Vasquez-Vasquez R, Otero C, Luna V, et al. (2016) Evaluation and optimization of occupational eye lens dosimetry during positron emission tomography (PET) procedures. J Radiol Prot 36: 299-308.

12. Carinou E, Ferrari P, Bjelac OC, Gingaume M, Sanns Merce M, et al. (2015) Eye lens monitoring for interventional radiology personnel: dosemeters, calibration and practical aspects of $\mathrm{Hp}(3)$ monitoring. A 2015 review. J Radiol Prot 35: R17-R34.

13. Strahlenschultzkommission (2015) Monitoring the eye lens dose. Recommendation of the German Commission on Radiological Protection.

14. Gualdrini G, Bordy JM, Daures J, Fantuzzi E, Ferrari P, et al. (2012) Air kerma to $\mathrm{Hp}(3)$ conversion coefficients for photons from $10 \mathrm{kev}$ to 10 mev, calculated in a cylindrical phantom. Radiat Prot dosimetry 154: 517-521.

15. Clairand I, Gijaume M, Vanhavere F, Carinou E, Daures J, et al. (2015) First Eurados intercomparison exercise of eye lens dosemeters for medical applications. Radiat Prot Dosimetry 368: 1-6.

16. Al-Haj AN, Lobriguito AM, Al Gain I (2015) Staff eye doses in a large medical centre in Saudi Arabia: are they meeting the new ICRP recommendations? Radiat Prot Dosimetry 165: 294-298.

17. Al-Haj AN, Lobriguito AM, Arafah A, Parker R (2010) Deriving staff and public doses in a PET/CT facility from measured radiation levels using thermoluminescent dosimetry. Radiat Prot Dosimetry 144: 487-491. 